\title{
CHEP 2021: Preface to the Proceedings
}

\author{
Catherine Biscarat ${ }^{1, *}$, Simone Campana ${ }^{2, * *}$, Benedikt Hegner $^{2, * * *}$, Stefan Roiser $^{2, * * * *}$, Chiara \\ I Rovelli ${ }^{3, \dagger}$, and Graeme A Stewart ${ }^{2, *}$ \\ ${ }^{1}$ L2I - Toulouse, CNRS and Univ. Paul Sabatier, Toulouse, France \\ ${ }^{2}$ CERN, 1 Esplanade des Particules, 1211 Geneva 23, Switzerland \\ ${ }^{3}$ Sapienza Universita e INFN, Roma I, Italy
}

\begin{abstract}
The $25^{\text {th }}$ International Conference on Computing in High Energy and Nuclear Physics (CHEP), organised by CERN, took place as a virtual event from 17-21 May 2021. The conference attracted 1144 registered participants from 46 different countries. There were 207 scientific presentations made over the 5 days of the conference. These were divided between 30 long talks and 2 keynotes, which were presented in plenary sessions; and 175 short talks, which were presented in parallel sessions.
\end{abstract}

\section{Foreword}

In May 2021 the $25^{\text {th }}$ International Conference on Computing in High Energy and Nuclear Physics (CHEP) was held. Since the last CHEP in Adelaide (November 2019), the world has been in the grip of the Covid-19 pandemic, which meant that the intended 2021 conference in Norfolk, Virginia, had to be postponed. Therefore, in late 2020, with the support of the CHEP International Advisory Committee, CERN agreed to organise a virtual edition of CHEP ( $v C H E P$ ), to afford the software and computing communities of nuclear and high energy physics (NHEP) the chance to present and publish their work.

The organisation of CHEP as a virtual event was quite a different challenge from that associated with a face-to-face CHEP so a number of important changes were made. Instead of a short abstract, submissions to the conference were full draft papers. While this is common practice in other fields it was the first time that this had been tried in this branch of NHEP. The authors of papers were asked to concentrate on specific areas of novel research and development and to identify a particular technological theme for their work, rather than organising by traditional CHEP tracks.

Despite the challenge of front-loading the work in submissions, more than 200 papers were received, underlining the appetite of researchers to participate in a CHEP, even one that was hosted virtually.

The full papers allowed the organisers to select a plenary programme that was based on the work submitted to the conference, rather than the normal plenary program of invited

\footnotetext{
*e-mail: catherine.biscarat@12it.in2p3.fr

**e-mail: simone.campana@cern.ch

***e-mail: benedikt.hegner@cern.ch

****e-mail: stefan.roiser@cern.ch

†e-mail: chiara.rovelli@cern.ch

†e-mail: graeme.andrew.stewart@cern.ch
} 


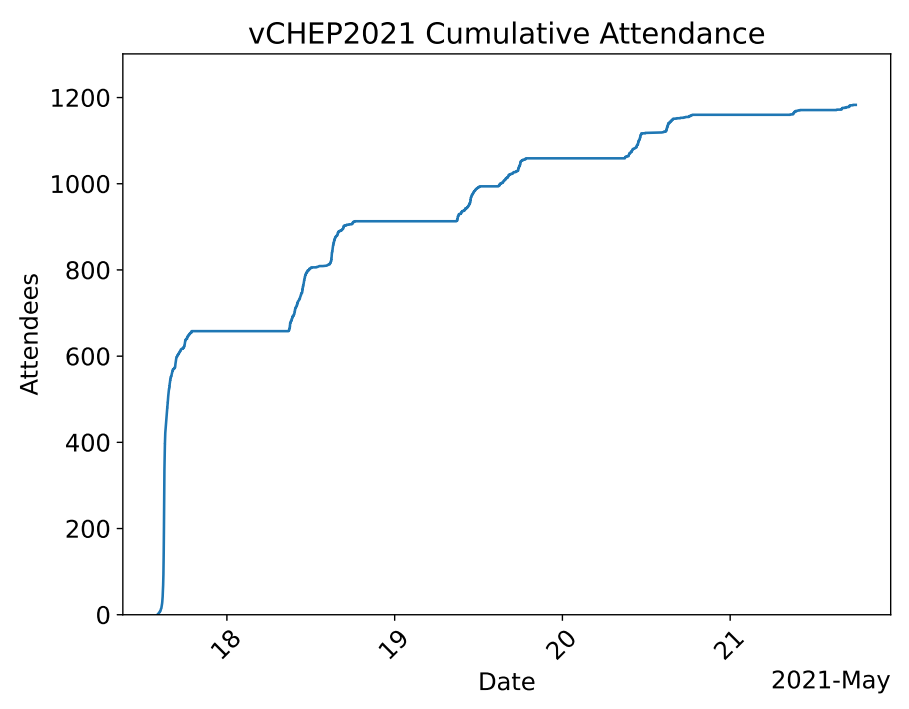

Figure 1. vCHEP cumulative attendance.

speakers, many from outside NHEP. This gave a unique focus to vCHEP, highlighting the excellent on-going work to tackle the upcoming challenges in the field.

In addition to the plenary programme, a number of parallel sessions were organised, which focused on particular technological themes, thus attracting a diverse set of papers from different parts of the NHEP workflow and being complimentary to a more traditional trackbased organisation.

As a virtual event, vCHEP had to strive for a schedule that would allow for participation from across the world - vCHEP was a truly global affair with participants from 46 countries and spanning 20 hours of timezones, from Brisbane (UTC+10) to Honolulu (UTC-10). The solution adopted was to have "morning" sessions (at least in the CERN timezone), adapted to Europe and Asia-Pacific, a long break, then "afternoon" sessions, adapted for Europe and the Americas. As it was clearly impossible to present at any time that was suitable for all participants, every talk was recorded and these were made available a few hours after the presentations and have also been archived via the conference website.

In addition to providing the live video conferencing sessions and the video recordings, for the first time at CHEP, a chat system was employed that allowed participants to continue discussions of papers offline, including from incompatible timezones.

As we have learned since the start of the pandemic, virtual events are treated as more of a buffet than a banquet, with participants free to join only the sessions that interest them. However, in total vCHEP recorded a cumulative participation of 1182 unique connections and largest single-session attendance of 496 people (Figure 1).

In addition to these conference proceedings a number of vCHEP papers are being prepared for fully-refereed publication in Computing and Software for Big Science (Springer), which has been appreciated as an option for the most outstanding contributions.

vCHEP was a success and exceeded the organisers and the IAC's expectations for an event restricted to be held virtually. However, we recognise that not all of the value of a normal CHEP could be offered at this exceptional vCHEP - the informal and face-to-face interactions, so valued by all of us, were missing. Therefore we hope very much that the next 
edition of CHEP, the re-scheduled event in Norfolk, Virginia, in May 2022, will see a return to a format that allows this very important aspect of the CHEP series to happen again.

\section{CHEP conference series}

The CHEP conference series was established in 1985, and since then has been one of the most important events in the field of computing in high energy and nuclear physics. The conference covers a broad set of topics such as online, offline and distributed computing; software development, simulation, reconstruction and analysis packages; data handling, databases and storage solutions; clouds, virtualisation and containers; networking and facilities, including high performance computing. This year, for the first time, a parallel session on quantum computing was organised. It provides a valuable discussion platform, enabling the exchange of ideas between physicists, computing scientists and software engineers, as well as between renowned experts and young researchers.

The CHEP conference is normally held every 18 months. The host location is selected on a rotating basis between the geographical regions of Europe, the Americas, and Asia-Pacific. The intention for the $25^{\text {th }}$ CHEP was to hold it in Norfolk, Virginia, USA. However, the global pandemic forced the plans for such a CHEP, with a traditional format, to be postponed for 12 months. The CHEP International Advisory Committee considered that a gap of 30 months between CHEPs was too long and would lose momentum for the conference series and lose a valuable opportunity for the community to present and discuss work, particularly for early career researchers. Bids were invited to organise an exceptional virtual edition of CHEP, and an offer from CERN to organise vCHEP was accepted.

The list of past CHEP conferences is shown in Table 1.

Table 1: Dates and locations of previous CHEP conferences.

\begin{tabular}{lll}
\hline Name & Dates & Location \\
\hline CHEP'85 & 25-28 June 1985 & Amsterdam, Netherlands \\
CHEP'87 & 2-6 February 1987 & Asilomar, California, USA \\
CHEP'89 & 10-14 April 1989 & Oxford, England, United Kingdom \\
CHEP'90 & 9-13 April 1990 & Santa Fe, New Mexico, USA \\
CHEP'91 & 11-15 March 1991 & Tsukuba, Japan \\
CHEP'92 & 21-25 September 1992 & Annecy, France \\
CHEP'94 & 21-27 April 1994 & San Francisco, California, USA \\
CHEP'95 & 18-22 September 1995 & Rio de Janeiro, Brazil \\
CHEP'97 & 7-11 April 1997 & Berlin, Germany \\
CHEP'98 & 31 August-4 September 1998 & Chicago, Illinois, United States \\
CHEP2000 & 7-11 February 2000 & Padova, Italy \\
CHEP'01 & 3-7 September 2001 & Beijing, China \\
CHEP'03 & 24-28 March 2003 & San Diego, California, USA \\
CHEP'04 & 27 September - 1 October 2004 & Interlaken, Switzerland \\
CHEP'06 & 13-17 February 2006 & Mumbai, India \\
CHEP'07 & 2-7 September 2007 & Victoria, British Columbia, Canada \\
CHEP'09 & 21-27 March 2009 & Prague, Czech Republic \\
CHEP'10 & 18-22 October 2010 & Taipei, Taiwan \\
CHEP'12 & 21-25 May 2012 & New York, New York, USA \\
CHEP'13 & 14-18 October 2013 & Amsterdam, Netherlands \\
CHEP'15 & 13-17 April 2015 & Okinawa, Japan \\
CHEP'16 & 10-14 October 2016 & San Francisco, California, USA
\end{tabular}




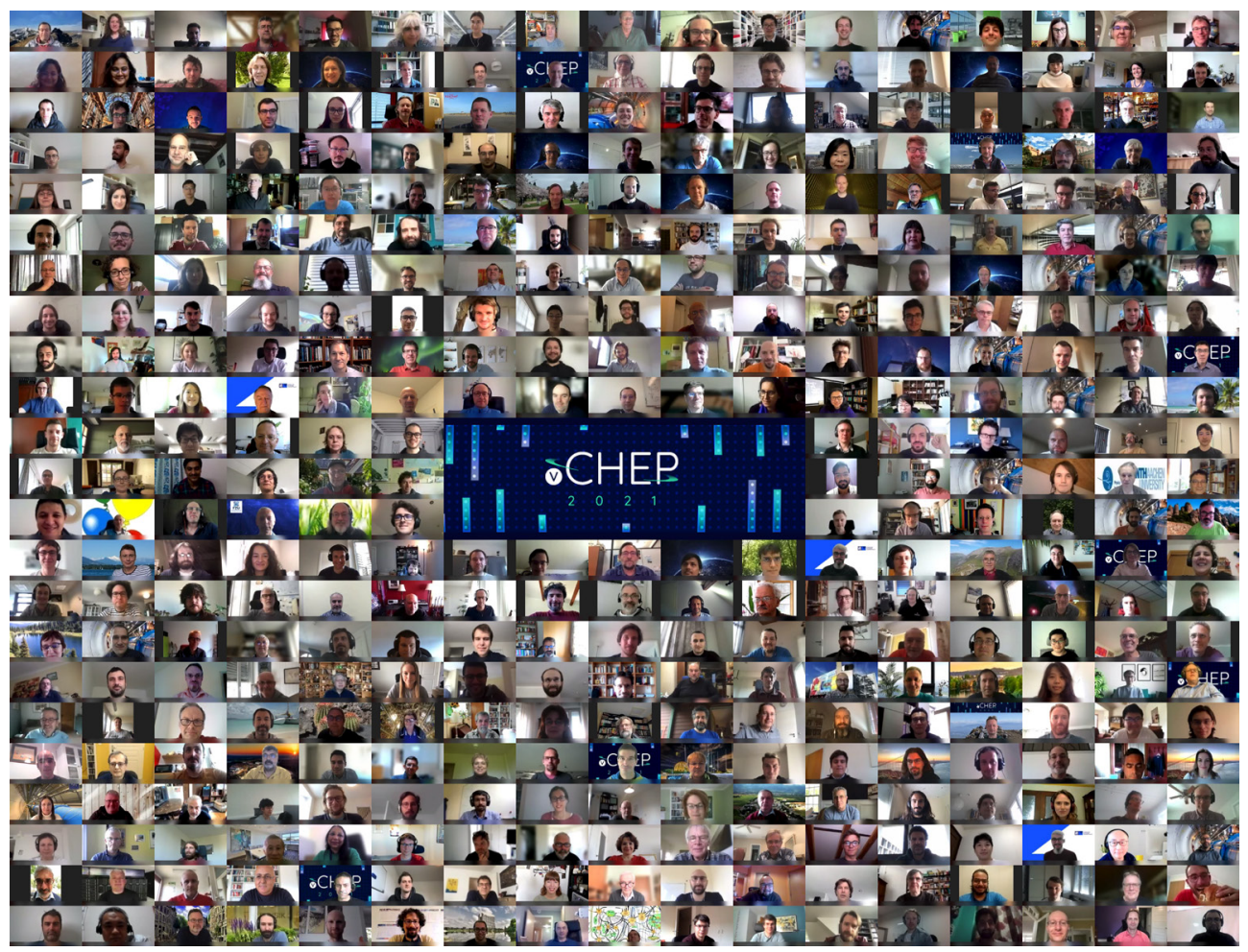

Figure 2. The vCHEP 2021 conference photo.

Table 1: Dates and locations of previous CHEP conferences.

\begin{tabular}{lll}
\hline Name & Dates & Location \\
\hline CHEP'18 & 9-13 July 2018 & Sofia, Bulgaria \\
CHEP'19 & 4-8 November 2019 & Adelaide, Australia \\
\hline
\end{tabular}

The (virtual) conference photograph for vCHEP 2021 is shown in Figure 2.

\section{CHEP 2021 Conference}

\subsection{Conference Programme}

In contradistinction to the usual process at CHEP, where a short abstract is submitted, usually to a specific parallel session track, for vCHEP authors were asked to submit a full draft paper of 6-10 pages. Despite the short timescale for writing such papers (barely three months between the call and the close of submissions, including the need for some experiment reviews), more than 200 papers were received.

When submitting, the number of traditional tracks was pared down from previous CHEPs to just four: Offline Computing; Distributed Computing, Data Management and Facilities; Online Computing; Collaboration, Education, Training and Outreach. The distribution of papers against these tracks is shown in Figure 3. 


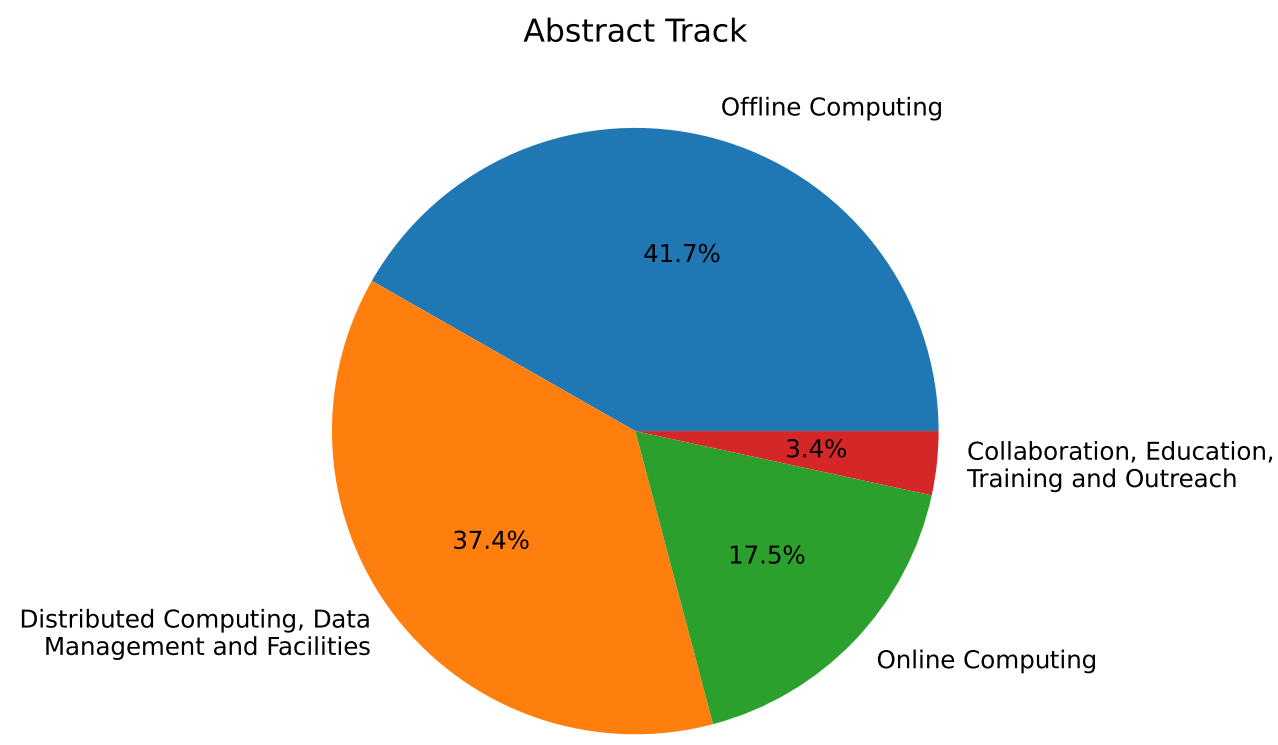

Figure 3. vCHEP paper submissions by track.

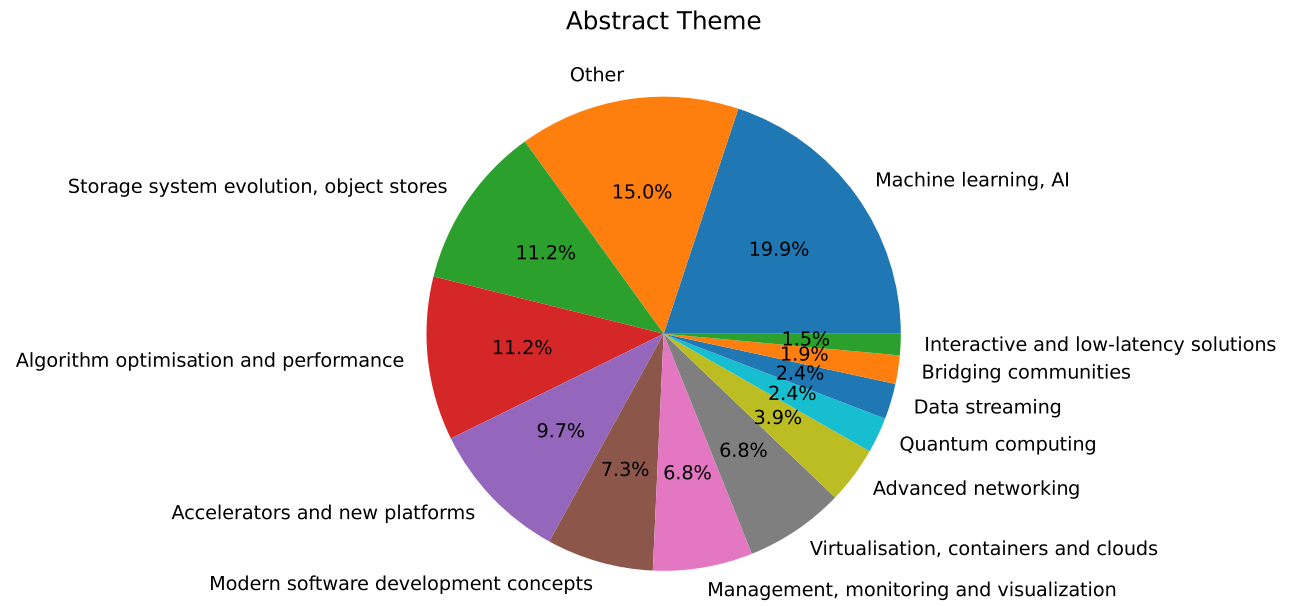

Figure 4. vCHEP paper submissions by themes.

In addition, authors could highlight a particular theme, which were organised on a more technological basis, that helped identify common trends in an orthogonal way to the tracks. The distribution of papers against themes is shown in Figure 4.

Due to the special nature of vCHEP it was decided that the plenary programme would be formed principally of the most innovative papers from the NHEP field itself. Thirty papers were selected from the submissions to form the plenary programme, from across all of 
the topics that were submitted. In addition, two keynotes were invited in order to give an overview of the computing and software domains in NHEP, which set the stage for the rest of the conference.

When organising the parallel sessions themes were chosen as the organising principle, to draw connections between areas that would not normally be grouped when organising by track. Of particular note is that at vCHEP the first parallel session on Quantum Computing was organised for the CHEP series.

\subsection{Programme Committee}

The CHEP 2021 Programme Committee (PC) members were charged with reviewing the submissions to vCHEP and providing feedback to the authors for preparing the final versions. We would like to thank them particularly for the work they managed to do in reviewing full papers on a very short timescale. The Programme Committee also helped to chair the parallel sessions.

The selection of papers for the plenary session was managed by the Conference and Programme Committee Chairs, based on inputs from the Programme Committee reviews.

The PC members are listed in Table 2.

Table 2: CHEP 2021 Programme Committee

\begin{tabular}{ll}
\hline Name & Affiliation \\
\hline Agnieszka Dziurda & Polish Academy of Sciences \\
Andrea Formica & Université Paris-Saclay \\
Andrea Sartirana & CNRS/IN2P3 \\
Andrea Sciaba & CERN \\
Ben Couturier & CERN \\
Benjamin Krikler & Bristol \\
Benedikt Hegner (PC Co-chair) & CERN \\
Caterina Doglioni & Lund \\
Catherine Biscarat (PC Co-chair) & L2I - Toulouse, CNRS / Univ. Paul Sabatier \\
Chiara Rovelli (PC Co-chair) & INFN Roma \\
Cedric Serfon & BNL \\
Christophe Haen & CERN \\
Clara Gaspar & CERN \\
Clara Nellist & NIKHEF \\
Daniela Bauer & Imperial London \\
Daniele Spiga & INFN Perugia \\
Dave Britton & Glasgow \\
David Bouvet & CNRS/CC-IN2P3 \\
David Crooks & RAL \\
David Rohr & CERN \\
Dirk Duellmann & CERN \\
Dmytro Kresan & GSI \\
Dorothea vom Bruch & CNRS/CPPM \\
Edoardo Martelli & CERN \\
Eduardo Rodrigues & Liverpool \\
Elizabeth Sexton-Kennedy & FNAL \\
Enrico Guiraud & CERN \\
Eric Yen & ASGC
\end{tabular}


Table 2: CHEP 2021 Programme Committee

\begin{tabular}{ll}
\hline Name & Affiliation \\
\hline Felice Pantaleo & CERN \\
Ghita Rahal & CNRS/CC-IN2P3 \\
Gian Michele Innocenti & CERN \\
Giulio Eulisse & CERN \\
Gonzalo Merino Arevalo & PIC \\
Gordon Watts & Washington \\
Graeme A Stewart & CERN \\
Jason Webb & BNL \\
Jerome Lauret & BNL \\
John Chapman & Cambridge \\
Joosep Pata & NICPB Estonia \\
Julia Andreeva & CERN \\
Katy Ellis & RAL \\
Latchezar Betev & CERN \\
Luisa Arrabito & CNRS/IN2P3 \\
Maarten Litmaath & CERN \\
Marc Dunser & CERN \\
Maria Girone & CERN \\
Markus Schulz & CERN \\
Marzena Lapka & CERN \\
Niko Neufeld & CERN \\
Peter Clarke & Edinburgh \\
Peter Hristov & CERN \\
Reda Tafirout & TRIUMF \\
Shawn McKee & Michigan \\
Simon George & RHUL \\
Simone Campana & CERN \\
Simone Pigazzini & ETH \\
Sofia Vallecorsa & CERN \\
Stefan Roiser (PC Co-chair) & CERN \\
Steve Timm & FNAL \\
Stewart Martin-Haugh & RAL \\
Takanori Hara & KEK \\
Teng Jian Khoo & Humboldt \\
Tommaso Boccali & INFN Pisa \\
Tomoe Kishimoto & Tokyo \\
Vardan Gyurjyan & JLab \\
Xavier Espinal & CERN \\
Yuji Kato & Nagoya \\
\hline & \\
& \\
& \\
\hline
\end{tabular}

\subsection{International Advisory Committee}

The CHEP 2021 International Advisory Committee provided essential strategic advice for the organisation of the event and helped to run the plenary sessions. The members of the IAC are listed in Table 3. The CHEP 2021 IAC met six times, including one meeting held after the conference itself. 
Table 3: CHEP 2021 International Advisory Committee

\begin{tabular}{|c|c|}
\hline Name & Affiliation \\
\hline Alessandra Forti & Manchester \\
\hline Amber Boehnlein & JLab \\
\hline Andreas Wicenec & UWA/ICRAR \\
\hline Benedikt Hegner (PC Co-chair) & CERN \\
\hline Bronson E Messer II & ORNL \\
\hline Caterina Doglioni & Lund \\
\hline Catherine Biscarat (PC Co-chair) & L2I - Toulouse, CNRS / Univ. Paul Sabatier \\
\hline Chiara Rovelli (PC Co-chair) & INFN Roma \\
\hline Chris Cuevas & $\mathrm{JLab}$ \\
\hline Concezio Bozzi & INFN Ferrara \\
\hline Danilo Piparo & CERN \\
\hline David Britton & Glasgow \\
\hline David Groep & NIKHEF \\
\hline Doris Kim & Soongsil \\
\hline Elizabeth Sexton-Kennedy & FNAL \\
\hline Eric Yen & ASGC \\
\hline Gang Chen & IHEP \\
\hline Ghita Rahal & CNRS/CC-IN2P3 \\
\hline Gonzalo Merino Arevalo & PIC \\
\hline Gordon Watts & Washington \\
\hline Graeme A Stewart (Conference Co-chair) & CERN \\
\hline Graham Heyes & JLab \\
\hline Hannah Short & CERN \\
\hline Heather Gray & LBNL \\
\hline Heidi Schellman & Oregon \\
\hline Ian Bird & CERN \\
\hline Ikuo Ueda & KEK \\
\hline James Catmore & Oslo \\
\hline Jerome Lauret & $\mathrm{BNL}$ \\
\hline Julia Andreeva & CERN \\
\hline Latchezar Betev & CERN \\
\hline Lucia Silvestris & INFN Bari \\
\hline Marco Cattaneo & CERN \\
\hline Maria Girone & CERN \\
\hline Michel Jouvin & IJCLab \\
\hline Mohammad Al-Turany & GSI \\
\hline Niko Neufeld & CERN \\
\hline Oxana Smirnova & Lund \\
\hline Pat Scott & Queensland \\
\hline Patrick Fuhrmann & DESY \\
\hline Paul Jackson & Adelaide \\
\hline Paul Laycock & BNL \\
\hline Pere Mato Vila & CERN \\
\hline Peter Clarke & Edinburgh \\
\hline Peter Elmer & Princeton \\
\hline Peter Hristov & CERN \\
\hline Raffaella Devita & JLab \\
\hline
\end{tabular}


Table 3: CHEP 2021 International Advisory Committee

\begin{tabular}{ll}
\hline Name & Affiliation \\
\hline Randy Sobie & Victoria \\
Reda Tafirout & TRIUMF \\
Richard Mount & SLAC \\
Sang-Un Ahn & KISTI \\
Simone Campana (Conference Co-chair) & CERN \\
Stefan Roiser (PC Co-chair) & CERN \\
Stefano Piano & INFN Trieste \\
Takanori Hara & KEK \\
Tommaso Boccali & INFN Pisa \\
Torre Wenaus & BNL \\
Waseem Kamleh & Adelaide \\
\hline
\end{tabular}

\subsection{Local Organisation}

The CHEP 2021 conference was chaired by Simone Campana and Graeme A Stewart. The chairs wish to thank the secretariat, Catherine Noble, for her tremendous work in ensuring the organisation of the conference ran smoothly. As a virtual conference we needed to have excellent IT support for running the conference website, managing submissions, hosting sessions, handling videos, etc. For this, invaluable assistance was provided by the members of the local organising committee, listed in Table 4.

Table 4: CHEP 2021 Local Organising Committee

\begin{tabular}{ll}
\hline Name & Affiliation \\
\hline Simone Campana (Conference Co-chair) & CERN \\
Graeme A Stewart (Conference Co-chair) & CERN \\
Catharine Noble (Secretariat) & CERN \\
Hanna Short & CERN \\
Helge Meinhard & CERN \\
Latchezar Betev & CERN \\
Maria Girone & CERN \\
Markus Elsing & CERN \\
Melissa Gaillard & CERN \\
Peter Hristov & CERN \\
Sebastian Lopienski & CERN \\
Thomas Baron & CERN \\
Adeel Ahmad & CERN \\
Julia Andreeva & CERN \\
Xavier Espinal & CERN \\
Eric Grancher & CERN \\
Viktor Khristenko & CERN \\
Maarten Litmaath & CERN \\
Edoardo Martelli & CERN \\
David Southwick & CERN \\
Eric Wulff & CERN \\
Anirudh Goel & CERN \\
Katarzyna Dziedziniewicz & CERN \\
\hline
\end{tabular}




\section{Acknowledgements}

The organisers would like to acknowledge the strong support of the CERN directorate for CHEP 2021. This allowed us to run a very successfully event, at no cost to participants, and to publish the proceedings and CSBS articles, at no cost to the authors.

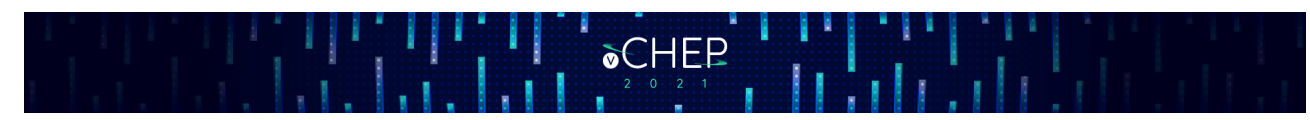

